\title{
Food-based diet quality score in relation to depressive symptoms in young and middle-aged Japanese women
}

\author{
Hiroka Sakai $^{1}$, Kentaro Murakami ${ }^{2 *}$, Satomi Kobayashi ${ }^{3}$, Hitomi Suga ${ }^{4}$, Satoshi Sasaki ${ }^{3}$ and \\ the Three-generation Study of Women on Diets and Health Study Group \\ ${ }^{1}$ Department of Nutrition, School of Human Cultures, University of Shiga Prefecture, Shiga 522 8533, Japan \\ ${ }^{2}$ Interfaculty Initiative in Information Studies, University of Tokyo, Tokyo 113 O033, Japan \\ ${ }^{3}$ Department of Social and Preventive Epidemiology, School of Public Health, University of Tokyo, Tokyo 113 O033, Japan \\ ${ }^{4}$ Department of Social and Preventive Epidemiology, Graduate School of Medicine, University of Tokyo, Tokyo 113 0033, Japan
}

(Submitted 23 February 2017 - Final revision received 30 May 2017 - Accepted 2 June 2017)

\section{Abstract}

Only a few studies have focused on the association between overall diet, rather than intakes of individual nutrients or foods, and depressive symptoms in Japanese. This cross-sectional study examined associations between a diet quality score and depressive symptoms in 3963 young (age 18 years) and 3833 middle-aged (mean age 47.9 (sD 4.2) years) Japanese women. Dietary information was collected using a diet history questionnaire. A previously developed diet quality score was computed mainly based on the Japanese Food Guide Spinning Top. The prevalence of depressive symptoms was $22.0 \%$ for young women and $16.8 \%$ for middle-aged women, assessed as a Center for Epidemiologic Studies Depression (CES-D) score $\geq 23$ and $\geq 19$, respectively. As expected, the diet quality score was associated positively with intakes of 'grain dishes', 'vegetable dishes', 'fish and meat dishes', 'milk' and 'fruits' and inversely with intakes of energy from 'snacks, confection and beverages' and $\mathrm{Na}$ from seasonings. After adjustment for potential confounders, OR for depressive symptoms in the highest $v$. lowest quintiles of the diet quality score was 0.65 (95\% CI $0.50,0.84)$ in young women $\left(P_{\text {for trend }}=0.0005\right)$. In middle-aged women, the corresponding value was $0.59(95 \%$ CI $0.45,0.78)\left(P_{\text {for trend }}<0.0001\right)$. Analyses where the diet quality and CES-D scores were treated as continuous variables also showed inverse associations. In conclusion, this cross-sectional study showed that a higher diet quality score was associated with a lower prevalence of depressive symptoms in young and middle-aged Japanese women. Prospective studies are needed to confirm a public health relevance of this finding.

Key words: Depressive symptoms: Japanese Food Guide Spinning Top: Diet quality score: Japanese women: Cross-sectional design

Depression is a common mental disorder ${ }^{(1)}$. In Japan, the number of patients of depression has been increasing ${ }^{(2)}$, although a crossnational analysis based on data from selected eighteen countries has shown that Japan is among the lowest for the prevalence of major depressive episodes ${ }^{(3)}$. Emerging evidence suggests that intakes of specific nutrients (such as folate ${ }^{(4,5)}$ and long-chain $n-3$ PUFA $^{(6,7)}$ ) and foods (such as fish ${ }^{(7-9)}$ and fruit and vegetable $\left.{ }^{(10,11)}\right)$ are associated with a lower risk of depressive symptoms. However, given that people eat foods and thus nutrients in combination, an inherent limitation of the study of single nutrients or foods in isolation is no consideration of complicated interactions and cumulative effects that individual dietary components involve ${ }^{(12)}$. Accordingly, focus has shifted in recent years towards overall diet and dietary patterns ${ }^{(13-16)}$.

Two main approaches are used to identify patterns of dietary intake. One is the a posteriori approach, which uses statistical techniques based on dietary intake reported by a population ${ }^{(17)}$. Another is the a priori approach, which uses diet quality scores or indices based on dietary guidelines ${ }^{(17)}$. Many Western studies have investigated associations between overall diet and depressive symptoms using either of the approaches. Generally, a healthy dietary pattern, characterised by higher intakes of fruit, vegetables, fish and whole grains, has been associated with a lower risk of depressive symptoms ${ }^{(18-24)}$.

The associations of dietary intake with depressive symptoms observed in Western populations may not be applicable to Japanese people, given differences in dietary habits. The diets consumed by Japanese are typically characterised by high intakes of rice, soya products, fish, seaweeds and green tea and low intakes of animal fat and soft drinks ${ }^{(25)}$. Actually, previous Japanese studies have shown inverse associations of intakes of fish $^{(8)}$, seaweed $^{(26)}$, soya products ${ }^{(27)}$ and green tea ${ }^{(28,29)}$ with

Abbreviations: CES-D, Center for Epidemiologic Studies Depression; DHQ, diet history questionnaire; EI, energy intake.

* Corresponding author: Dr K. Murakami, fax +81 35841 7873, email kenmrkm@m.u-tokyo.ac.jp 
the prevalence of depressive symptoms. To our knowledge, however, only a few studies have focused on the association between dietary patterns and depressive symptoms in Japanese ${ }^{(30-32)}$. Furthermore, the findings from these studies are not readily applicable to, for example, the development of dietary recommendations or public health messages because of the use of data-driven approach for dietary patterns.

We previously developed a diet quality score mainly based on the Japanese Food Guide Spinning Top, using intakes of 'grain dishes', 'vegetable dishes', 'fish and meat dishes', 'milk', 'fruits', 'snacks, confection and beverages' and seasonings ${ }^{(33)}$. Although depressive symptoms were not specifically considered in its development, the diet quality score was associated with nutrient intake patterns possibly favourable for the prevention of depressive symptoms ${ }^{(33)}$, including lower intakes of total and saturated fats ${ }^{(8)}$ and higher intakes of dietary fibre ${ }^{(34)}, \mathrm{Ca}^{(35)}$, $\mathrm{Mg}^{(36,37)}, \mathrm{Fe}^{(38)}$, folate $\mathrm{e}^{(4,5)}$ and vitamin $\mathrm{C}^{(39)}$. Therefore, the aim of this cross-sectional study in young and middle-aged Japanese women was to examine the hypothesis that a higher diet quality score is associated with a lower prevalence of depressive symptoms.

\section{Methods}

\section{Survey design and analytic sample}

This cross-sectional study was based on data obtained from the Three-generation Study of Women on Diets and Health, details of which have been described elsewhere ${ }^{(33,40)}$. In brief, a total of 7016 Japanese dietetic students from eighty-five higher education institutions in thirty-five (of forty-seven) prefectures in Japan were asked to answer two questionnaires on dietary habits and other lifestyle factors during the orientation session or first lecture designed for freshmen in April 2011 or 2012. Each student was also asked to directly distribute the questionnaires to his or her mother (and grandmother or female acquaintance aged 65-89 years) and invite them to participate in the study. A total of 4933 students (including 4656 women and 277 men; response rate: $70 \cdot 3 \%$ ), 4044 mothers (response rate: $57 \cdot 6 \%$ ) and 2332 women from grandmother's generation (response rate: $33.2 \%$ ) answered both questionnaires. The present study was conducted according to the guidelines laid down in the Declaration of Helsinki and all procedures involving human subjects were approved by the Ethics Committee of the University of Tokyo Faculty of Medicine. Written informed consent was obtained from each participant, and also from a parent for participants aged $<20$ years.

In the present study, we selected female students aged 18 years $(n$ 4065) and all mothers ( $n$ 4044) for the analyses of young and middle-aged women, respectively; we did not use data from grandmother's generation mainly because of the use of different dietary assessment method as well as a low response rate. We then excluded students who answered the questionnaires more than 1 month after the start of the course to minimise the influence of dietetic education ( $n$ 56); subjects living in eastern Japan who participated in the 2011 data collection period, because it was assumed that they could not report their usual dietary habits and lifestyle because of the occurrence of the Great East Japan Earthquake in March 2011 ( $n 39$ and 63 for young and middle-aged women, respectively); and those from one institution at which the response rate was extremely low ( $2 \% ; n 2$ for both young and middle-aged women). We further excluded those with missing information on the variables of interest ( $n 5$ and 146 for young and middleaged women, respectively). Consequently, the final analysis sample sizes were 3963 and 3833 for young and middle-aged women, respectively.

\section{Dietary assessment}

Dietary habits during the preceding month were assessed using a comprehensive diet history questionnaire (DHQ). Responses to the DHQ and also to the lifestyle questionnaire were checked by survey staff at the study centre. If any missing or erroneous responses were given to questions considered essential for analysis, the subject was asked to complete the questions again. Details of the DHQ's structure and calculation method of dietary intake have been published elsewhere ${ }^{(41-43)}$. In brief, the DHQ is a structured, self-administered questionnaire which asks about the consumption frequency and portion size of selected foods commonly consumed in Japan, as well as general dietary behaviour and usual cooking methods. Estimates of daily intake of foods (151 items in total), energy and selected nutrients were calculated using an ad boc computer algorithm for the DHQ based on the Standard Tables of Food Composition in Japan ${ }^{(44)}$. To minimise the influence of dietary misreporting ${ }^{(45,46)}$, energy-adjusted values of dietary intake based on the density method were used (i.e. percentage of energy for energy-providing nutrients and amount per $4184 \mathrm{~kJ}$ of energy for foods and other nutrients). The validity of the DHQ was examined in ninety-two women aged 31-69 years using a 16-d weighed dietary record as reference ${ }^{(41,42)}$. In brief, the median of Spearman's correlation coefficients for food groups was 0.43 (range: -0.09-0.77), and the median of Pearson's correlation coefficients for nutrients was 0.57 (range: $0 \cdot 27-0 \cdot 87$ ).

\section{Calculation of diet quality score}

Using dietary information derived from the DHQ, a previously developed diet quality score was computed. The calculation method for the diet quality score has been described in detail elsewhere $^{(33)}$ and in the online Supplementary Table S1. In brief, the diet quality score (which is described as 'the modified score' in the previous publication ${ }^{(33)}$ ) is based on six components recommended in the Japanese Food Guide Spinning Top as well as $\mathrm{Na}$ from seasonings (seven components in total). When intake was within the recommended range, a score of 10 was assigned to that component. Energy-adjusted values of dietary intake based on the density method were calculated to obtain the values per $7531 \mathrm{~kJ}$ of energy to allow comparison with the recommended values. The recommended range was as follows: $\geq 4$ servings/7531 kJ for 'grain dishes', $\geq 5$ servings/ $7531 \mathrm{~kJ}$ for 'vegetable dishes', $\geq 3$ servings/7531 kJ for 'fish and meat dishes', $\geq 2$ servings/7531 kJ for 'milk', $\geq 2$ servings/7531 kJ for 'fruits', $\leq 837 \mathrm{~kJ} / 7531 \mathrm{~kJ}$ for energy from 'snacks, confection and beverages' and $\leq 10$ th percentile (i.e. $2619 \mathrm{mg} / 7531 \mathrm{~kJ}$ for 
young women and $3108 \mathrm{mg} / 7531 \mathrm{~kJ}$ for middle-aged women) for $\mathrm{Na}$ from seasonings. For a subject who fell short of or exceeded the recommended value, the score was calculated proportionately between 0 and 10 (the scoring system is shown in the online Supplementary Table S1). The seven scores were then summed to provide the diet quality score, which ranged from 0 to 70 .

\section{Assessment of depressive symptoms}

Depressive symptoms were assessed using the Japanese version $^{(47)}$ of the Center for Epidemiologic Studies Depression (CES-D) scale ${ }^{(48)}$. This scale is composed of twenty items addressing six symptoms of depression, including depressed mood, guilt or worthlessness, helplessness or hopelessness, psychomotor retardation, loss of appetite and sleep disturbance experienced during the preceding week. Each item was scored on a scale of 0-3 according to the frequency of the symptom and total CES-D score was calculated, which ranged from 0 to 60 . The criterion validity of the CES-D scale has been well established in Western $^{(48)}$ and Japanese ${ }^{(47)}$ subjects. Because of a previously demonstrated over-rating of symptoms by the Japanese $\mathrm{u}^{(49,50)}$, particularly younger people ${ }^{(51,52)}$, prevalent cases of depressive symptoms were defined as having a CES-D score of $\geq 23^{(49)}$ for young women and $\geq 19^{(49)}$ for middle-aged women, rather than a widely used cutoff point of $\geq 16^{(47,48)}$. The use of the cutoff points $\geq 16$ for both age groups, $\geq 19$ for young women and $\geq 23$ for middle-aged women did not change the conclusion on the association between the diet quality score and depressive symptoms (data not shown).

\section{Assessment of other variables}

Age at the time of the study was computed based on birth date. BMI was computed as self-reported body weight $(\mathrm{kg})$ divided by the square of self-reported body height (m). Information on current smoking and use of any type of prescribed medicine (both yes or no) was also used. Physical activity was computed as the total metabolic equivalent-hours score per $\mathrm{d}$ on the basis of the frequency and duration of seven activities (walking, bicycling, standing, running, high-intensity activities, sleeping and sedentary activity) over the preceding month ${ }^{(53)}$. For middle-aged women only, education level (low ( $\leq 12$ years), middle (13-15 years) or high ( $\geq 16$ years)), occupation (housewife, part-time job or full-time job), and marital status (married or unmarried) were considered. Living alone (yes or no) was considered for young women, but not for middle-age women because almost all lived with family. Self-reported level of stress was classified as very low, low, normal, high or very high. Dietary reporting status was evaluated on the basis of the ratio of reported energy intake (EI):BMR (Goldberg's cut-off) ${ }^{(54)}$. A detailed description of the procedure has been published elsewhere ${ }^{(33)}$. In brief, BMR was estimated according to an equation specifically developed for Japanese women ${ }^{(55,56)}$. Subjects were identified as plausible, under- and over-reporters of EI according to whether the individual's ratio was within, below or above the $95 \%$ confidence limits for agreement between EI:BMR and a physical activity level for sedentary lifestyle (i.e. 1.55$)^{(54)}$. As a result, under-reporters, plausible reporters and over-reporters were defined as having an EI:BMR $<1.09,1.09-2.21$ and $>2.21$, respectively.

\section{Statistical analysis}

All statistical analyses were performed using SAS statistical software version 9.4 (SAS Institute Inc.). All reported $P$ values are two-tailed, and $P<0.05$ was regarded as statistically significant. We decided a priori to conduct analyses for young and middle-aged women separately, mainly because of the differences in potential confounding factors that should be considered. We did not conduct any formal tests for interaction across age groups. Descriptive data are shown as means and standard deviations for continuous variables and numbers and percentages of subjects for categorical variables. Differences in characteristics between subjects with and without depressive symptoms were evaluated using an independent $t$ test (for continuous variables) and the $\chi^{2}$ test (for categorical variables). The diet quality score was categorised at quintile points on the basis of the distribution of young and middle-aged women. Associations between selected variables and diet quality score (in quintiles) were examined by a linear trend test (for continuous variables) and a Mantel-Haenszel $\chi^{2}$ test (for categorical variables).

Crude and multivariate adjusted OR and 95\% CI for depressive symptoms for each quintile category of the diet quality score were calculated using logistic regression. The lowest quintile category of diet quality score was used as a reference category. Potential confounding factors considered were BMI, current smoking, medication use, self-reported level of stress, dietary reporting status, physical activity and EI. Adjustment for EI was due to a significant difference in EI between subjects with and without depressive symptoms (as described in Table 1), despite the use of energy-adjusted values for the calculation of the diet quality score. Further adjustment was made for living status in young women and age, education, occupation and marital status in middle-aged women. Further adjustment for the presence of child's depressive symptoms in the analysis of middle-aged women and that of mother's depressive symptoms in the analysis of young women did not change the results materially (data not shown). We tested for linear trends with increasing levels of diet quality score by assigning each subject the median value for the category and modelling this value as a continuous variable. These analyses were repeated after excluding under- and over-reporters of EI.

\section{Results}

All of the young women were 18 years old while mean age of the middle-aged women was 47.9 (SD 4.2) years. The prevalence of depressive symptoms was $22.0 \%$ for young women and $16.8 \%$ for middle-aged women. Young and middle-aged women with depressive symptoms were more likely to use medication and have higher levels of stress and had a higher mean value of EI and a lower mean value of the diet quality score (Table 1). In addition, middle-aged women with 
Table 1. Basic characteristics of young and middle-aged Japanese women (Mean values and standard deviations; percentages)

\begin{tabular}{|c|c|c|c|c|c|c|c|c|c|c|c|c|c|c|}
\hline & \multicolumn{7}{|c|}{ Young } & \multicolumn{7}{|c|}{ Middle-aged } \\
\hline & \multicolumn{2}{|c|}{ All ( $n$ 3963) } & \multicolumn{2}{|c|}{$\begin{array}{l}\text { Subjects with } \\
\text { depressive } \\
\text { symptoms } \\
(n 871)^{*}\end{array}$} & \multicolumn{2}{|c|}{$\begin{array}{c}\text { Subjects } \\
\text { without } \\
\text { depressive } \\
\text { symptoms } \\
\text { (n 3092)† }\end{array}$} & \multirow[b]{2}{*}{$P \ddagger$} & \multicolumn{2}{|c|}{ All $(n$ 3833) } & \multicolumn{2}{|c|}{$\begin{array}{l}\text { Subjects with } \\
\text { depressive } \\
\text { symptoms } \\
(n \text { 643) }\end{array}$} & \multicolumn{2}{|c|}{$\begin{array}{c}\text { Subjects } \\
\text { without } \\
\text { depressive } \\
\text { symptoms } \\
\text { (n 3190)† }\end{array}$} & \multirow[b]{2}{*}{$P \ddagger$} \\
\hline & Mean & SD & Mean & $\mathrm{SD}$ & Mean & SD & & Mean & $\mathrm{SD}$ & Mean & SD & Mean & $\mathrm{SD}$ & \\
\hline BMI $\left(\mathrm{kg} / \mathrm{m}^{2}\right)$ & $20 \cdot 9$ & $2 \cdot 8$ & $21 \cdot 1$ & $3 \cdot 2$ & $20 \cdot 8$ & $2 \cdot 7$ & 0.01 & $22 \cdot 1$ & $3 \cdot 1$ & $22 \cdot 1$ & 3.3 & $22 \cdot 0$ & $3 \cdot 1$ & 0.58 \\
\hline Current smoking (\%) & & & & & & & 0.67 & & & & & & & 0.005 \\
\hline Yes & \multicolumn{2}{|c|}{$0 \cdot 2$} & \multicolumn{2}{|c|}{0.2} & \multicolumn{2}{|c|}{0.2} & & \multicolumn{2}{|c|}{$7 \cdot 6$} & \multicolumn{2}{|c|}{$10 \cdot 3$} & \multicolumn{2}{|c|}{$7 \cdot 0$} & \\
\hline No & \multicolumn{2}{|c|}{99.8} & \multicolumn{2}{|c|}{$99 \cdot 8$} & \multicolumn{2}{|c|}{$99 \cdot 8$} & & \multicolumn{2}{|c|}{92.4} & \multicolumn{2}{|c|}{$89 \cdot 7$} & \multicolumn{2}{|c|}{93.0} & \\
\hline Medication use (\%) & \multirow{2}{*}{\multicolumn{2}{|c|}{$10 \cdot 8$}} & & & & & 0.006 & & & & & & & 0.0006 \\
\hline Yes & & & \multicolumn{2}{|c|}{$13 \cdot 3$} & \multicolumn{2}{|c|}{$10 \cdot 1$} & & \multicolumn{2}{|c|}{$25 \cdot 4$} & \multicolumn{2}{|c|}{$30 \cdot 8$} & \multicolumn{2}{|c|}{$24 \cdot 4$} & \\
\hline No & \multicolumn{2}{|c|}{$89 \cdot 2$} & 86 & .7 & \multicolumn{2}{|c|}{89.9} & & 74 & & 69 & 9.2 & 75 & 6 & \\
\hline Self-reported level of stress (\%) & & & & & & & $<0.0001$ & & & & & & & $<0.0001$ \\
\hline Very low & & 1.3 & & $\cdot 2$ & & 5.4 & & & 1.3 & & 3.6 & & .4 & \\
\hline Low & & 9.0 & & .0 & 11 & $1 \cdot 2$ & & & 9.2 & & 5.6 & & 9 & \\
\hline Normal & 59 & 9.1 & 53 & 3 & 65 & 5.6 & & 59 & & 56 & $6 \cdot 1$ & 59 & 6 & \\
\hline High & 24 & 4.9 & 36 & 1 & 17 & 7.0 & & 24 & 1.8 & 29 & 9.9 & 23 & 3.8 & \\
\hline Very high & & 2.8 & & 4 & & .9 & & & 2.7 & & 4.8 & & 3 & \\
\hline Dietary reporting status (\%)§ & & & & & & & 0.06 & & & & & & & 0.048 \\
\hline Under-reporting & 18 & 3.7 & 19 & 4 & 18 & 3.5 & & & 7.7 & & $7 \cdot 8$ & & 7.7 & \\
\hline Plausible reporting & 74 & $\cdot 7$ & 72 & .5 & 75 & 5.4 & & 81 & 1.0 & 77 & $7 \cdot 8$ & 81 & 1.7 & \\
\hline Over-reporting & & 5 & & $3 \cdot 2$ & & $5 \cdot 1$ & & 11 & 1.3 & 14 & 4.5 & 10 & .7 & \\
\hline $\begin{array}{l}\text { Physical activity (total metabolic } \\
\text { equivalent-h/d) }\end{array}$ & $37 \cdot 8$ & 5.5 & $38 \cdot 0$ & $5 \cdot 9$ & $37 \cdot 8$ & $5 \cdot 4$ & 0.28 & $40 \cdot 7$ & $5 \cdot 6$ & $39 \cdot 8$ & $5 \cdot 7$ & 40.9 & 5.5 & $<0.0001$ \\
\hline $\mathrm{EI}(\mathrm{kJ} / \mathrm{d})$ & 7242 & 2399 & 7434 & 2804 & 7187 & 2269 & 0.007 & 7714 & 2229 & 7912 & 2607 & 7673 & 2142 & 0.01 \\
\hline Diet quality scorell & $41 \cdot 1$ & $7 \cdot 9$ & $39 \cdot 8$ & $8 \cdot 1$ & 41.4 & $7 \cdot 8$ & $<0.0001$ & 42.9 & $8 \cdot 2$ & 41.3 & 8.5 & 43.2 & $8 \cdot 1$ & $<0.0001$ \\
\hline
\end{tabular}

El, energy intake.

* Subjects with a Center for Epidemiologic Studies Depression score $\geq 23$ for young women and $\geq 19$ for middle-aged women.

† Subjects with a Center for Epidemiologic Studies Depression score $<23$ for young women and $<19$ for middle-aged women.

$\ddagger P$ values for difference between subjects with and without depressive symptoms based on the independent $t$ test for continuous variables and the $\chi^{2}$ test for categorical variables. $\S$ Under-reporting was defined as subjects with a ratio of reported El:BMR <1.09; plausible reporting as an El:BMR of 1.09-2.21; and over-reporting as an El:BMR >2.21.

II The diet quality score (0-70) was developed based on the Japanese Food Guide Spinning Top with some modifications, and calculated using intakes of 'grain dishes', 'vegetable dishes', 'fish and meat dishes', 'milk', 'fruits', energy from 'snacks, confection and beverages' and Na from seasonings (see online Supplementary Table S1 for more details).

depressive symptoms were more likely to be younger, have lower education and be unmarried (data not shown).

As expected, the diet quality score was associated positively with intakes of 'grain dishes', 'vegetable dishes', 'fish and meat dishes', 'milk' and 'fruits' and inversely with intakes of energy from 'snacks, confection and beverages' and $\mathrm{Na}$ from seasonings in both age groups (Table 2). For food group level, a higher diet quality score was characterised particularly by higher intakes of dairy products, fruit, seaweed, soya products and vegetables and lower intakes of confectioneries, sugar and soft drinks (online Supplementary Table S2). For nutrient intakes, the diet quality score was associated inversely with total and saturated fats and positively with EPA + DHA, dietary fibre and micronutrients examined (online Supplementary Table S3). There were significant associations between the diet quality score and most of the potential confounding factors considered in both young and middle-aged women (online Supplementary Tables S4 and S5, respectively).

Table 3 shows the association between the diet quality score and depressive symptoms. In young women, with adjustment for potential confounding factors (i.e. BMI, current smoking, medication use, self-reported level of stress, dietary reporting status, physical activity, EI and living alone), a higher diet quality score was associated with a lower prevalence of depressive symptoms. A similar inverse association was also observed in middle-aged women after adjustment for potential confounding factors (i.e. age, education, occupation, marital status and the variables listed above except for living alone). After excluding under- and over-reporters of EI ( $n 1001$ for young women and $n 728$ for middle-aged women), the inverse associations did remain in both age groups. Further, the analysis where both the diet quality score and CES-D score were treated as continuous variables did not change the conclusion (online Supplementary Table S6).

\section{Discussion}

To our knowledge, this is the first study to examine a diet quality score, mainly based on the Japanese Food Guide Spinning Top, using intakes of 'grain dishes', 'vegetable dishes', 'fish and meat dishes', 'milk', 'fruits', energy from 'snacks, confection and beverages' and $\mathrm{Na}$ from seasonings, in relation to depressive symptoms in young and middle-aged women from a variety of regions in Japan. We found that a higher diet quality score, characterised particularly by higher intakes of dairy products, fruit, seaweed, soya products, vegetables, $\mathrm{EPA}+\mathrm{DHA}$, dietary fibre, $\mathrm{Ca}, \mathrm{Mg}, \mathrm{Fe}$, folate and vitamin $\mathrm{C}$ and lower intakes of confectioneries, sugar, soft 
Table 2. Descriptive statistics of the diet quality score in young and middle-aged Japanese women* (Mean values and standard deviations)

\begin{tabular}{|c|c|c|c|c|c|c|c|c|c|c|c|c|c|}
\hline & \multicolumn{2}{|c|}{ All } & \multicolumn{2}{|c|}{ Quintile 1} & \multicolumn{2}{|c|}{ Quintile 2} & \multicolumn{2}{|c|}{ Quintile 3} & \multicolumn{2}{|c|}{ Quintile 4} & \multicolumn{2}{|c|}{ Quintile 5} & \multirow[b]{2}{*}{$P_{\text {for trend }} \dagger$} \\
\hline & Mean & SD & Mean & SD & Mean & SD & Mean & SD & Mean & SD & Mean & SD & \\
\hline Young & \multicolumn{2}{|c|}{$n 3963$} & \multicolumn{2}{|c|}{$n 792$} & \multicolumn{2}{|c|}{$n 793$} & \multicolumn{2}{|c|}{$n 793$} & \multicolumn{2}{|c|}{$n 793$} & \multicolumn{2}{|c|}{$n 792$} & \\
\hline Median diet quality score & & & 30 & & 36 & & 41 & & 45 & & 51. & & \\
\hline $\begin{array}{l}\text { Score range } \\
\text { Score componentsł }\end{array}$ & \multicolumn{2}{|c|}{$14.50-67.73$} & \multicolumn{2}{|c|}{$14.50-34.21$} & \multicolumn{2}{|c|}{$34.22-38.91$} & \multicolumn{2}{|c|}{$38.92-43 \cdot 10$} & \multicolumn{2}{|c|}{$43 \cdot 11-47 \cdot 97$} & \multicolumn{2}{|c|}{$47 \cdot 98-67 \cdot 73$} & \\
\hline Grain dishes (servings) & 3.8 & $1 \cdot 1$ & 3.3 & $1 \cdot 1$ & 3.7 & $1 \cdot 2$ & 3.9 & $1 \cdot 2$ & 3.9 & $1 \cdot 1$ & 4.0 & 1.0 & $<0.0001$ \\
\hline Vege & 3.6 & $2 \cdot 2$ & $2 \cdot 7$ & 1.7 & 3.2 & 1.8 & 3.7 & $2 \cdot 2$ & 3.9 & $2 \cdot 2$ & 4.5 & $2 \cdot 4$ & $<0$. \\
\hline eat dishes (servings) & 4.7 & $1 \cdot 8$ & 4.5 & 1.9 & 4.6 & $1 \cdot 7$ & 4.7 & $1 \cdot 8$ & $5 \cdot 0$ & 1.9 & 4.7 & 1.6 & $<0.0001$ \\
\hline Milk (servings) & 1.3 & 1.4 & 0.5 & 0.6 & 0.9 & $1 \cdot 1$ & 1.3 & 1.5 & 1.7 & 1.5 & $2 \cdot 3$ & 1.6 & $<0.0001$ \\
\hline Fruits & 0.7 & 0.8 & 0.4 & 0.5 & 0.5 & 0.5 & 0.6 & 0.8 & 0.8 & 0.9 & $1 \cdot 1$ & 0.9 & $<0.0001$ \\
\hline $\begin{array}{l}\text { Energy from snacks, confection and } \\
\text { beverages (kJ) }\end{array}$ & 1322 & 686 & 1996 & 669 & 1536 & 627 & 1243 & 569 & 1000 & 431 & 837 & 388 & $<0.0001$ \\
\hline $\mathrm{Na}$ from seasonings $(\mathrm{mg})$ & 2075 & 984 & 2232 & 996 & 2102 & 917 & 2110 & 1008 & 2126 & 1071 & 1807 & 858 & $<0.0001$ \\
\hline Middle-aged & \multirow{2}{*}{\multicolumn{2}{|c|}{$\begin{array}{c}n 3833 \\
42.9\end{array}$}} & \multirow{2}{*}{\multicolumn{2}{|c|}{$\begin{array}{c}n 766 \\
32 \cdot 1\end{array}$}} & \multirow{2}{*}{\multicolumn{2}{|c|}{$\begin{array}{c}n 767 \\
38.4\end{array}$}} & \multirow{2}{*}{\multicolumn{2}{|c|}{$\begin{array}{c}n 767 \\
42.9\end{array}$}} & \multicolumn{2}{|c|}{$n 767$} & \multicolumn{2}{|c|}{$n 766$} & \\
\hline Median diet qua & & & & & & & & & 47 & & \multirow{2}{*}{\multicolumn{2}{|c|}{$\begin{array}{c}53.7 \\
50.03-69.53\end{array}$}} & \\
\hline $\begin{array}{l}\text { Score range } \\
\text { Score compon }\end{array}$ & \multicolumn{2}{|c|}{$14.24-69.53$} & \multicolumn{2}{|c|}{$14 \cdot 24-35 \cdot 68$} & \multicolumn{2}{|c|}{$35 \cdot 69-40 \cdot 84$} & \multicolumn{2}{|c|}{$40 \cdot 85-45 \cdot 22$} & \multicolumn{2}{|c|}{$45 \cdot 23-50 \cdot 02$} & & & \\
\hline Grair & 3.5 & 1.0 & $3 \cdot 0$ & 0.9 & 3.3 & 1.0 & 3.6 & $1 \cdot 0$ & 3.6 & 1.0 & 3.7 & 0.9 & $<0.0001$ \\
\hline hes (servings) & 3.9 & $2 \cdot 0$ & $3 \cdot 2$ & 1.7 & 3.6 & 1.7 & 3.9 & 1.8 & 4.2 & $2 \cdot 1$ & 4.7 & $2 \cdot 1$ & $<0.0001$ \\
\hline Fish a & $5 \cdot 1$ & 1.6 & 4.9 & 1.7 & $5 \cdot 0$ & 1.6 & $5 \cdot 1$ & 1.6 & $5 \cdot 3$ & 1.6 & $5 \cdot 0$ & 1.4 & 0.04 \\
\hline Milk & 1.5 & 1.4 & 0.6 & 0.7 & 1.1 & 1.1 & 1.5 & 1.3 & 1.9 & 1.4 & 2.5 & 1.5 & $<0.0001$ \\
\hline Fruits (servings) & 0.7 & 0.6 & 0.3 & 0.4 & 0.5 & 0.4 & 0.6 & 0.6 & 0.8 & 0.7 & $1 \cdot 1$ & 0.8 & $<0.0001$ \\
\hline $\begin{array}{l}\text { Energy from snacks, confection and } \\
\text { beverages (kJ) }\end{array}$ & 1314 & 636 & 1941 & 607 & 1527 & 577 & 1268 & 523 & 1017 & 414 & 816 & 314 & $<0.0001$ \\
\hline $\mathrm{Na}$ from seasonings $(\mathrm{mg})$ & 2205 & 945 & 2480 & 1008 & 2236 & 909 & 2201 & 992 & 2146 & 909 & 1945 & 787 & $<0.0001$ \\
\hline
\end{tabular}

* The diet quality score (0-70) was developed based on the Japanese Food Guide Spinning Top with some modifications, and calculated using intakes of 'grain dishes', 'vegetable dishes', 'fish and meat dishes', 'milk', 'fruits', energy from 'snacks, confection and beverages' and Na from seasonings (see online Supplementary Table S1 for more details). $\dagger \mathrm{A}$ liner trend test was used with the median value in each quintile category of the diet quality score as a continuous variable in linear regression.

$\ddagger$ Per $7531 \mathrm{~kJ}$ of energy.

Table 3. Associations between the diet quality score and depressive symptoms in young and middle-aged Japanese women* (Odds ratios and $95 \%$ confidence intervals)

\begin{tabular}{|c|c|c|c|c|c|c|c|c|c|c|c|c|c|c|}
\hline & \multicolumn{7}{|c|}{ All } & \multicolumn{7}{|c|}{ Only subjects reporting plausible energy intake† } \\
\hline & \multirow[b]{2}{*}{$n$} & \multirow{2}{*}{$\begin{array}{l}\text { Median } \\
\text { diet quality } \\
\text { score }\end{array}$} & \multirow{2}{*}{$\begin{array}{c}\text { Depressive } \\
\text { symptoms (\%)§ }\end{array}$} & \multicolumn{2}{|c|}{ Crude model } & \multicolumn{2}{|c|}{$\begin{array}{l}\text { Multivariate } \\
\text { model } \ddagger\end{array}$} & \multirow[b]{2}{*}{$n$} & \multirow{2}{*}{$\begin{array}{c}\text { Median } \\
\text { diet quality } \\
\text { score }\end{array}$} & \multirow{2}{*}{$\begin{array}{c}\text { Depressive } \\
\text { symptoms }(\%) \S\end{array}$} & \multicolumn{2}{|c|}{ Crude model } & \multicolumn{2}{|c|}{$\begin{array}{l}\text { Multivariate } \\
\text { model } \ddagger\end{array}$} \\
\hline & & & & OR & $95 \% \mathrm{Cl}$ & OR & $95 \% \mathrm{Cl}$ & & & & OR & $95 \% \mathrm{Cl}$ & OR & $95 \% \mathrm{Cl}$ \\
\hline Young & 3963 & & & & & & & 2962 & & & & & & \\
\hline Quintile 1 & 792 & $30 \cdot 7$ & $27 \cdot 8$ & 1 & Ref. & 1 & Ref. & 592 & $30 \cdot 7$ & $27 \cdot 2$ & 1 & Ref. & 1 & Ref. \\
\hline Quintile 2 & 793 & $36 \cdot 7$ & 23.8 & 0.81 & $0.65,1.02$ & 0.91 & $0.70,1.17$ & 593 & $36 \cdot 7$ & $23 \cdot 1$ & 0.80 & $0.62,1.05$ & 0.93 & $0.70,1.25$ \\
\hline Quintile 3 & 793 & $41 \cdot 1$ & $19 \cdot 4$ & 0.63 & $0.50,0.79$ & 0.73 & $0.56,0.95$ & 592 & $41 \cdot 3$ & $18 \cdot 6$ & 0.61 & $0.46,0.80$ & 0.68 & $0.50,0.93$ \\
\hline Quintile 4 & 793 & $45 \cdot 3$ & $20 \cdot 2$ & 0.66 & $0.52,0.83$ & 0.76 & $0.59,0.99$ & 593 & 45.5 & $20 \cdot 1$ & 0.67 & $0.51,0.88$ & 0.78 & $0.57,1.05$ \\
\hline $\begin{array}{l}\text { Quintile } 5 \\
P_{\text {for trend }} \|\end{array}$ & 792 & $51 \cdot 3$ & $18 \cdot 7$ & 0.60 & $\begin{array}{l}0.47,0.76 \\
0.0001\end{array}$ & $\begin{array}{r}0.65 \\
0\end{array}$ & $\begin{array}{l}0.50,0.84 \\
0.0005\end{array}$ & 592 & 51.4 & $17 \cdot 6$ & 0.57 & $\begin{array}{l}0.43,0.75 \\
0.0001\end{array}$ & 0.67 & $\begin{array}{l}0.49,0.92 \\
0.005\end{array}$ \\
\hline Middle-aged & 3833 & & & & & & & 3105 & & & & & & \\
\hline Quintile 1 & 766 & $32 \cdot 1$ & $22 \cdot 7$ & 1 & Ref. & 1 & Ref. & 621 & 32.5 & 21.9 & 1 & Ref. & 1 & Ref. \\
\hline Quintile 2 & 767 & 38.4 & $18 \cdot 1$ & 0.75 & $0.59,0.97$ & 0.80 & $0.62,1.03$ & 621 & 38.7 & $18 \cdot 0$ & 0.79 & $0.59,1.04$ & 0.82 & $0.62,1.09$ \\
\hline Quintile 3 & 767 & 42.9 & $15 \cdot 3$ & 0.61 & $0.47,0.79$ & 0.67 & $0.51,0.87$ & 621 & $43 \cdot 2$ & 14.0 & 0.58 & $0.43,0.78$ & 0.60 & $0.45,0.82$ \\
\hline Quintile 4 & 767 & 47.5 & $14 \cdot 2$ & 0.56 & $0.43,0.73$ & 0.62 & $0.47,0.81$ & 621 & $47 \cdot 8$ & $13 \cdot 7$ & 0.57 & $0.42,0.76$ & 0.61 & $0.45,0.83$ \\
\hline Quintile 5 & 766 & $53 \cdot 7$ & $13 \cdot \overline{6}$ & 0.54 & $0.41,0.70$ & 0.59 & $0.45,0.78$ & 621 & $53 \cdot 8$ & $12 \cdot 9$ & 0.53 & $0.39,0.71$ & 0.55 & $0.40,0.75$ \\
\hline$P_{\text {for trendll }}$ & & & & & 0.0001 & & 0.0001 & & & & & 0.0001 & & $<0.0001$ \\
\hline
\end{tabular}

Ref., reference.

* The diet quality score (0-70) was developed based on the Japanese Food Guide Spinning Top with some modifications, and calculated using intakes of 'grain dishes', 'vegetable dishes', 'fish and meat dishes', 'milk', 'fruits', energy from 'snacks, confection and beverages' and Na from seasonings (see online Supplementary Table S1 for more details). $\dagger$ Plausible energy intake reporters were defined as subjects with a ratio of energy intake:BMR of 1.09-2.21.

$\ddagger$ Adjustment was made for BMI ( $\mathrm{kg} / \mathrm{m}^{2}$, continuous), current smoking (yes or no), medication use (yes or no), self-reported level of stress (very low, low, normal, high or very high), dietary reporting status (under-reporting, plausible reporting or over-reporting; only in the analysis including all subjects), physical activity (total metabolic equivalent-h/d, continuous) and energy intake (kJ/d, continuous). For young women, additional adjustment was made for living alone (yes or no). For middle-aged women, additional adjustment was made for age (years, continuous), education (low, middle or high), occupation (housewife, part-time job or full-time job) and marital status (married or unmarried).

$\S$ Depressive symptoms were defined as present when subjects had a Center for Epidemiologic Studies Depression score $\geq 23$ for young women and $\geq 19$ for middle-aged women.

II Logistic regression models were used with the median value in each quintile category of the diet quality score as a continuous variable in logistic regression.

drinks and total and saturated fats, was associated with a lower prevalence of depressive symptoms.

Our findings are generally consistent with previous Western studies $^{(18-24)}$ in that a healthy dietary pattern is inversely associated with depressive symptoms. In this study, however, a higher diet quality score was characterised by higher intakes of, for example, seaweed and soya products, foods seldom consumed in Western countries. Thus, healthy diets are country 
specific, and their health effects should be investigated in each country.

Only a few studies have examined associations between dietary patterns assessed by a data-driven statistical approach and depressive symptoms in Japanese. In a cross-sectional study in 309 men and 212 women aged 21-67 years, a healthy Japanese dietary pattern characterised by higher intakes of vegetables, fruit, mushrooms and soya products was associated with a lower prevalence of depressive symptoms ${ }^{(32)}$. Another cross-sectional study in 1792 men and 214 women aged 19-69 years showed that a dietary pattern characterised by higher intakes of folate, vitamin $\mathrm{C}, \mathrm{Mg}, \mathrm{Ca}, \mathrm{Fe}$ and $\mathrm{Zn}$ was associated with a lower prevalence of depressive symptoms ${ }^{(30)}$. These findings are generally consistent with the present observation. Conversely, a prospective study showed no clear associations of a healthy pattern (characterised by higher intakes of vegetables, seaweed, mushrooms, soya products, potatoes, fish and fruits and lower intakes of soft drinks and confectioneries) or a traditional Japanese pattern (characterised by higher intakes of rice, miso soup and pickled vegetables and lower intakes of bread, confectioneries and dairy products) during pregnancy with the risk of postpartum depression in 865 women (mean age: 29.9 (SD 4.0) years) $)^{(31)}$. The discrepant findings may be because of the differences in the population and the outcome examined, as well as the study design.

A range of individual dietary components may have a potential role on the development or prevention of depression. For example, decreased intake of $\mathrm{B}$ vitamins, particularly folate, may result in the accumulation of homocysteine and in a decreased synthesis of monoamines in the brain, likely contributing to mechanisms related to the origins of depression ${ }^{(57,58)}$. In addition, long-chain n-3 PUFA, particularly EPA and DHA, may play an important role in neurotransmitter synthesis, degradation, release, reuptake and binding, resulting in a pattern of neurotransmitter activity that has been associated with depression ${ }^{(59,60)}$. In this study, the diet quality score was positively associated with the intakes of specific dietary components whose higher intakes were previously suggested to lower the risk of depressive symptoms. These include dairy products $^{(35,61)}$, fruit ${ }^{(10,11)}$, seaweed ${ }^{(26)}$, soya products ${ }^{(27)}$, vegetables $^{(10,11)}, \mathrm{EPA}+\mathrm{DHA}^{(6,7)}$, dietary fibre ${ }^{(34)}, \mathrm{Ca}^{(35)}, \mathrm{Mg}^{(36,37)}$, $\mathrm{Fe}^{(38)}$, folate $\mathrm{e}^{(4,5)}$ and vitamin $\mathrm{C}^{(39)}$. The score was also inversely associated with intakes of confectioneries and sugar (as a source of added sugar ${ }^{(34)}$, soft drinks ${ }^{(62,63)}$ and total and saturated fats ${ }^{(8)}$, and higher intakes of these foods and nutrients have been suggested to increase the risk. Overall or cumulative effects derived from these individual dietary components may explain the association between the diet score and depressive symptoms observed in this study.

Several limitations of the present study should be acknowledged. First, because exposure and outcome were assessed at the same time, the results of this cross-sectional study are susceptible to reverse causation. As a result, we cannot exclude the possibility that depressive symptoms may lead to a lower diet quality. The relationship between dietary intake and mental health is complex and likely bidirectional, and the temporal direction of the association between diet quality and depressive symptoms could be both ways ${ }^{(64)}$. In any case, only a prospective study would provide better understanding of the relationship between diet quality and depressive symptoms.

Second, given the proportion of Japanese adolescents who study in college or university $(57 \%)^{(65)}$, our subjects (i.e. dietetic students and their mothers) are likely to have a relatively high socio-economic status. Further, dietetic students may be more conscious of their diet than the general population, whereas the present study was conducted in most institutions within 1 month after the dietetic course began to minimise the influence of dietetic education. In addition, the response rate was not high in mothers (57.6\%) compared with students (70.3\%), which may have caused self-selection bias. Therefore, our results might not be applicable to the general Japanese population.

Third, depressive symptoms were assessed by using the CES-D rather than a clinically administered structured diagnostic interview. CES-D is not a comprehensive psychiatric assessment. The absence of a clinical diagnosis may have led to the inclusion of subjects with chronic fatigue syndrome or atypical depression, resulting in an overestimation of the prevalence of depressive symptoms. Nevertheless, the prevalence of depressive symptoms assessed as a CES-D score $\geq 16$ in the present populations ( $50 \%$ for young women and $27 \%$ for middle-aged women) was generally comparable with that observed in undergraduate students $(50 \%)^{(52)}$ and in a representative sample of women aged $30-59$ years $(31 \%)^{(50)}$.

Fourth, all self-reported dietary assessment methods are susceptible to both random and systematic measurement errors $^{(45)}$. To minimise these, we evaluated dietary habits during the preceding month using a well-established dietary assessment questionnaire with reasonable validity in terms of commonly studied nutritional factors (DHQ) ${ }^{(41-43)}$, as well as the use of energy-adjusted dietary variables ${ }^{(45,46)}$. However, reporting bias in dietary questionnaires can differ by type of foods and therefore cannot be entirely adjusted for by the energy adjustment. Nevertheless, it should be noted that the present results did not change materially after excluding underand over-reporters of EI. Finally, although we tried to adjust for a range of potential confounding factors, residual confounding factors could not be ruled out.

In conclusion, this cross-sectional study showed that a higher diet quality score, mainly characterised by higher intakes of dairy products, fruit, seaweed, soya products, vegetables, $\mathrm{EPA}+\mathrm{DHA}$, dietary fibre, $\mathrm{Ca}, \mathrm{Mg}, \mathrm{Fe}$, folate and vitamin $\mathrm{C}$ and lower intakes of confectioneries, sugar, soft drinks and total and saturated fats, was associated with a lower prevalence of depressive symptoms in young and middle-aged Japanese women. A diet quality score of $\geq 39$ for young women and $\geq 41$ for middle-aged women (i.e. those in quintiles $3-5$ of the score) may be preferable for the prevention of depressive symptoms. Prospective studies are needed to confirm a public health relevance of the present finding.

\section{Acknowledgements}

The authors thank Yuki Kataya and Naoko Minobe (Department of Nutrition, School of Human Cultures, University of Shiga Prefecture, Japan) for assistance with manuscript preparation. The authors also 
thank the members of the Three-generation Study of Women on Diets and Health Study Group, as listed elsewhere ${ }^{(40)}$.

This work was supported in part by the Grants-in Aid for Young Scientists (to K. M., grant no. 15K16213) from the Ministry of Education, Culture, Sports, Science and Technology of Japan and the Grants-in-Aid for Scientific Research (to S. S., grant no. 22240077) from the Japan Society for the Promotion of Science. The Ministry of Education, Culture, Sports, Science and Technology of Japan and the Japan Society for the Promotion of Science had no role in the design, analysis or writing of this article.

H. S. contributed to the conceptualisation of the study question, conducted the statistical analysis and prepared the first draft of the manuscript; K. M. contributed to the concept and design of the survey, conceptualised the study question, interpreted the data, provided input into the final draft of the manuscript, prepared the revised version of the manuscript and had primary responsibility for the final content of the manuscript; S. K., H. S. and S. S. contributed to the concept and design of the survey, coordination of the fieldwork, data collection and management, and provided input into the final draft of the manuscript. All authors read and approved the final manuscript.

The authors declare that there are no conflicts of interest.

\section{Supplementary material}

For supplementary material/s referred to in this article, please visit https://doi.org/10.1017/S0007114517001581

\section{References}

1. Doris A, Ebmeier K \& Shajahan P (1999) Depressive illness. Lancet 354, 1369-1375.

2. Ministry of Education, Culture, Sports, Science and Technology (2014) Patient survey. http://www.mhlw.go.jp/toukei/ list/10-20.html (accessed April 2017) (in Japanese).

3. Bromet E, Andrade LH, Hwang I, et al. (2011) Cross-national epidemiology of DSM-IV major depressive episode. BMC Med 9, 90 .

4. Tolmunen T, Hintikka J, Ruusunen A, et al. (2004) Dietary folate and the risk of depression in Finnish middle-aged men. A prospective follow-up study. Psychother Psychosom 73, 334-339.

5. Murakami K, Mizoue T, Sasaki S, et al. (2008) Dietary intake of folate, other B vitamins, and $\omega-3$ polyunsaturated fatty acids in relation to depressive symptoms in Japanese adults. Nutrition 24, 140-147.

6. Colangelo LA, He K, Whooley MA, et al. (2009) Higher dietary intake of long-chain $\omega$-3 polyunsaturated fatty acid is inversely associated with depressive symptoms in women. Nutrition 25, 1011-1019.

7. Grosso G, Micek A, Marventano S, et al. (2016) Dietary n-3 PUFA, fish consumption and depression: a systematic review and meta-analysis of observational studies. I Affect Disord 205, 269-281.

8. Miyake $\mathrm{Y}$, Tanaka $\mathrm{K}$, Okubo $\mathrm{H}$, et al. (2013) Fish and fat intake and prevalence of depressive symptoms during pregnancy in Japan: baseline data from the Kyushu Okinawa Maternal and Child Health Study. J Psychiatr Res $\mathbf{4 7}$, 572-578.

9. Li F, Liu X \& Zhang D (2016) Fish consumption and risk of depression: a meta-analysis. J Epidemiol Community Health 70, 299-304.
10. Mihrshahi S, Dobson AJ \& Mishra GD (2015) Fruit and vegetable consumption and prevalence and incidence of depressive symptoms in mid-age women: results from the Australian longitudinal study on women's health. Eur J Clin Nutr 69, 585-591.

11. Kingsbury M, Dupuis G, Jacka F, et al. (2016) Associations between fruit and vegetable consumption and depressive symptoms: evidence from a national Canadian longitudinal survey. J Epidemiol Community Health 70, 155-161.

12. Hu FB (2002) Dietary pattern analysis: a new direction in nutritional epidemiology. Curr Opin Lipidol 13, 3-9.

13. Lai JS, Hiles S, Bisquera A, et al. (2014) A systematic review and meta-analysis of dietary patterns and depression in community-dwelling adults. Am J Clin Nutr 99, 181-197.

14. Quirk SE, Williams LJ, O'Neil A, et al. (2013) The association between diet quality, dietary patterns and depression in adults: a systematic review. BMC Psychiatry 13, 175.

15. Rahe C, Unrath M \& Berger K (2014) Dietary patterns and the risk of depression in adults: a systematic review of observational studies. Eur J Nutr 53, 997-1013.

16. Sanhueza C, Ryan L \& Foxcroft DR (2013) Diet and the risk of unipolar depression in adults: systematic review of cohort studies. J Hum Nutr Diet 26, 56-70.

17. Ocke MC (2013) Evaluation of methodologies for assessing the overall diet: dietary quality scores and dietary pattern analysis. Proc Nutr Soc 72, 191-199.

18. Jacka FN, Mykletun A, Berk M, et al. (2011) The association between habitual diet quality and the common mental disorders in community-dwelling adults: the Hordaland Health study. Psychosom Med 73, 483-490.

19. Akbaraly TN, Brunner EJ, Ferrie JE, et al. (2009) Dietary pattern and depressive symptoms in middle age. Br J Psychiatry 195, 408-413.

20. Samieri C, Jutand MA, Feart C, et al. (2008) Dietary patterns derived by hybrid clustering method in older people: association with cognition, mood, and self-rated health. J Am Diet Assoc 108, 1461-1471.

21. Rienks J, Dobson AJ \& Mishra GD (2012) Mediterranean dietary pattern and prevalence and incidence of depressive symptoms in mid-aged women: results from a large community-based prospective study. Eur J Clin Nutr 67, 75-82.

22. Akbaraly TN, Sabia S, Shipley MJ, et al. (2013) Adherence to healthy dietary guidelines and future depressive symptoms: evidence for sex differentials in the Whitehall II study. Am J Clin Nutr 97, 419-427.

23. Sanchez-Villegas A, Henriquez-Sanchez P, Ruiz-Canela M, et al. (2015) A longitudinal analysis of diet quality scores and the risk of incident depression in the SUN Project. BMC Med 13, 197.

24. Lai JS, Hure AJ, Oldmeadow C, et al. (2015) Prospective study on the association between diet quality and depression in mid-aged women over 9 years. Eur J Nutr 15, 1-9.

25. Ministry of Health, Labour and Welfare, Japan (2014) The National Health and Nutrition Survey in Japan, 2012. http:// www.mhlw.go.jp/bunya/kenkou/eiyou/h24-houkoku.html (accessed January 2017) (in Japanese).

26. Miyake Y, Tanaka K, Okubo H, et al. (2014) Seaweed consumption and prevalence of depressive symptoms during pregnancy in Japan: Baseline data from the Kyushu Okinawa Maternal and Child Health Study. BMC Pregnancy Childbirth 14, 301.

27. Miyake Y, Tanaka K, Okubo H, et al. (2016) Soy isoflavone intake and prevalence of depressive symptoms during pregnancy in Japan: baseline data from the Kyushu Okinawa Maternal and Child Health Study. Eur J Nutr (Epublication ahead of print version 15 October 2016). 
28. Niu K, Hozawa A, Kuriyama S, et al. (2009) Green tea consumption is associated with depressive symptoms in the elderly. Am J Clin Nutr 90, 1615-1622.

29. Pham NM, Nanri A, Kurotani K, et al. (2014) Green tea and coffee consumption is inversely associated with depressive symptoms in a Japanese working population. Public Health Nutr 17, 625-633.

30. Miki T, Kochi T, Kuwahara K, et al. (2015) Dietary patterns derived by reduced rank regression (RRR) and depressive symptoms in Japanese employees: The Furukawa nutrition and health study. Psychiatry Res 229, 214-219.

31. Okubo H, Miyake Y, Sasaki S, et al. (2011) Dietary patterns during pregnancy and the risk of postpartum depression in Japan: the Osaka Maternal and Child Health Study. Br J Nutr 105, 1251-1257.

32. Nanri A, Kimura Y, Matsushita Y, et al. (2010) Dietary patterns and depressive symptoms among Japanese men and women. Eur J Clin Nutr 64, 832-839.

33. Kuriyama N, Murakami K, Livingstone MB, et al. (2016) Development of a food-based diet quality score for Japanese: associations of the score with nutrient intakes in young, middle-aged, and older Japanese women. J Nutr Sci 5, e41.

34. Gangwisch JE, Hale L, Garcia L, et al. (2015) High glycemic index diet as a risk factor for depression: analyses from the Women's Health Initiative. Am J Clin Nutr 102, 454-463.

35. Miyake Y, Tanaka K, Okubo H, et al. (2015) Intake of dairy products and calcium and prevalence of depressive symptoms during pregnancy in Japan: a cross-sectional study. BJOG 122, 336-343.

36. Jacka FN, Overland S, Stewart R, et al. (2009) Association between magnesium intake and depression and anxiety in community-dwelling adults: the Hordaland Health Study. Aust $N$ Z J Psychiatry 43, 45-52

37. Jacka FN, Maes M, Pasco JA, et al. (2012) Nutrient intakes and the common mental disorders in women. J Affect Disord 141, 79-85.

38. Woo J, Lynn H, Lau WY, et al. (2006) Nutrient intake and psychological health in an elderly Chinese population. Int J Geriatr Psychiatry 21, 1036-1043.

39. Oishi J, Doi H \& Kawakami N (2009) Nutrition and depressive symptoms in community-dwelling elderly persons in Japan. Acta Med Okayama 63, 9-17.

40. Kobayashi S, Asakura K, Suga H, et al. (2013) High protein intake is associated with low prevalence of frailty among old Japanese women: a multicenter cross-sectional study. Nutr J 12, 164.

41. Kobayashi S, Honda S, Murakami K, et al. (2012) Both comprehensive and brief self-administered diet history questionnaires satisfactorily rank nutrient intakes in Japanese adults. J Epidemiol 22, 151-159.

42. Kobayashi S, Murakami K, Sasaki S, et al. (2011) Comparison of relative validity for food group intake estimated by comprehensive and brief-type self-administered diet history questionnaires against $16 \mathrm{~d}$ dietary records in Japanese adults. Public Health Nutr 14, 1200-1211.

43. Sasaki S, Yanagibori R \& Amano K (1998) Self-administered diet history questionnaire developed for health education: a relative validation of the test-version by comparison with 3-day diet record in women. J Epidemiol 8, 203-215.

44. Science and Technology Agency (2005) Standard Tables of Food Composition in Japan, Fatty Acid Section, 5 th rev. and enlarged ed. Tokyo: Printing Bureau of the Ministry of Finance (in Japanese).

45. Livingstone MB \& Black AE (2003) Markers of the validity of reported energy intake. J Nutr 133, 895S-920S.
46. Murakami K, Sasaki S, Uenishi K, et al. (2012) The degree of misreporting of the energy-adjusted intake of protein, potassium, and sodium does not differ among under-, acceptable, and over-reporters of energy intake. Nutr Res 32, 741-750.

47. Shima S, Shikano T, Kitamura T, et al. (1985) New self-rating scale for depression. Clin Psychiatry 27, 717-723 (in Japanese).

48. Radloff LS (1977) The CES-D scale: a self-report depression scale for research in the general population. Appl Psychol Meas 1, 385-401.

49. Wada K, Tanaka K, Theriault G, et al. (2007) Validity of the Center for Epidemiologic Studies Depression Scale as a screening instrument of major depressive disorder among Japanese workers. Am J Ind Med 50, 8-12.

50. Nagase Y, Uchiyama M, Kaneita Y, et al. (2009) Coping strategies and their correlates with depression in the Japanese general population. Psychiatry Res 168, 57-66.

51. Iwata N, Saito K \& Roberts RE (1994) Responses to a selfadministered depression scale among younger adolescents in Japan. Psychiatry Res 53, 275-287.

52. Hamazaki K, Natori T, Kurihara S, et al. (2015) Fish consumption and depressive symptoms in undergraduate students: a crosssectional analysis. Eur Psychiatry 30, 983-987.

53. Ainsworth BE, Haskell WL, Herrmann SD, et al. (2011) 2011 Compendium of physical activities: a second update of codes and MET values. Med Sci Sports Exerc 43, 1575-1581.

54. Black AE (2000) Critical evaluation of energy intake using the Goldberg cut-off for energy intake:basal metabolic rate. A practical guide to its calculation, use and limitations. Int J Obes Relat Metab Disord 24, 1119-1130.

55. Ganpule AA, Tanaka S, Ishikawa-Takata K, et al. (2007) Interindividual variability in sleeping metabolic rate in Japanese subjects. Eur J Clin Nutr 61, 1256-1261.

56. Miyake R, Tanaka S, Ohkawara K, et al. (2011) Validity of predictive equations for basal metabolic rate in Japanese adults. J Nutr Sci Vitaminol (Tokyo) 57, 224-232.

57. Bjelland I, Ueland PM \& Vollset SE (2003) Folate and depression. Psychother Psychosom 72, 59-60.

58. Gilbody S, Lightfoot $\mathrm{T} \&$ Sheldon T (2007) Is low folate a risk factor for depression? A meta-analysis and exploration of heterogeneity. J Epidemiol Community Health 61, 631-637.

59. Freeman MP (2000) Omega-3 fatty acids in psychiatry: a review. Ann Clin Psychiatry 12, 159-165.

60. Appleton KM, Rogers PJ \& Ness AR (2008) Is there a role for $n-3$ long-chain polyunsaturated fatty acids in the regulation of mood and behaviour? A review of the evidence to date from epidemiological studies, clinical studies and intervention trials. Nutr Res Rev 21, 13-41.

61. Cui Y, Huang C, Momma H, et al. (2017) Consumption of low-fat dairy, but not whole-fat dairy, is inversely associated with depressive symptoms in Japanese adults. Soc Psychiatry Psychiatr Epidemiol (Epublication ahead of print version 9 January 2017).

62. Yu B, He H, Zhang Q, et al. (2015) Soft drink consumption is associated with depressive symptoms among adults in China. J Affect Disord 172, 422-427.

63. Shi Z, Taylor AW, Wittert G, et al. (2010) Soft drink consumption and mental health problems among adults in Australia. Public Health Nutr 13, 1073-1079.

64. Jacka FN, Cherbuin N, Anstey KJ, et al. (2015) Does reverse causality explain the relationship between diet and depression? J Affect Disord 175, 248-250.

65. Ministry of Education, Culture, Sports, Science and Technology (2014) General survey of school. http://www.mext.go.jp/ component/b_menu/houdou/_icsFiles/afieldfile/2014/08/07/ 1350732_01.pdf (accessed January 2017) (in Japanese). 